\title{
CRESCIMENTO ECONÔMICO: UMA ANÁLISE DOS DETERMINANTES PARA OS BRICS NO PERÍODO DE 2000 A 2016
}

Francisco Dietima da Silva Bezerra ${ }^{1}$ Ariana Cericatto da Silva ${ }^{2}$

Resumo: O artigo tem como objetivo analisar os determinantes do crescimento econômico dos BRICS (Brasil, Rússia, Índia, China e África do Sul) para o período de 2000 a 2016. Utiliza-se do instrumental econométrico de Vetores Autorregressivos (VAR) e da Análise de Decomposição da Variância (ADV). Os resultados mostram que o investimento, a taxa de câmbio real efetiva e a inflação têm predominância na explicação do crescimento econômico dos países. Nesse sentido, uma gestão comprometida com o investimento e a estabilidade macroeconômica é crucial para se obter crescimento econômico elevado e sustentado no longo prazo.

Palavras-chave: Crescimento econômico. Análise de Decomposição da Variância (ADV). BRICS.

\section{ECONOMIC GROWTH: AN ANALYSIS OF THE DETERMINANTS FOR THE BRICS IN THE PERIOD 2000 TO 2016}

The article aims to analyze the determinants of the economic growth of the BRICS (Brazil, Russia, India, China and South Africa) for the period from 2000 to 2016. It used the econometric instruments of vectors autoregressive (VAR) and the variance decomposition analysis (VDA). The results show that investment, the real effective exchange rate and the inflation are predominant in explaining countries economic growth. In this sense, a management committed to investment and macroeconomic stability is crucial to obtain high and sustained economic growth in the long run.

Keywords: Economic growth. Variance Decomposition Analysis (VDA). BRICS.

\section{Introdução}

Com a intensificação do processo de globalização da economia, evidenciam-se diversas transformações econômicas e sociais em todo o mundo, dentre as quais destacam-se as elevadas taxas de crescimento das economias denominadas de "emergentes". Em estudo intitulado “Building Better Global Economic BRICs”, Jim O’Neill (2001) afirmou que o Brasil, a Rússia, a Índia e a China cresceriam a taxas superiores àquelas observadas nos países desenvolvidos. Essas

\footnotetext{
${ }^{1}$ Doutorando em Economia pela Universidade Federal de Uberlândia (UFU). Professor do Instituto Federal do Acre (IFAC). E-mail: dietimabezerra@yahoo.com.br

${ }^{2}$ Doutoranda em Economia pela Universidade Federal de Uberlândia (UFU). E-mail: ariana_cericatto@hotmail.com
} 
constatações foram cruciais para que, em 2006, esses países passassem a atuar na busca de uma política externa e comercial mais próxima, o que levou a criação do BRIC. Em 2011, a África do Sul foi incorporada ao grupo que, a partir de então, passou a ser denominado de BRICS (Brasil, Rússia, Índia, China e África do Sul) ${ }^{3}$.

Esses países que representavam cerca de 8,0\% do produto interno bruto (PIB) mundial em 2003, obtiveram uma participação de 18,0\% em 2010, portanto, um acréscimo significativo no decorrer desse período (WORLD BANK, 2018). De certo modo, a taxa de crescimento econômico cada vez mais elevada contribuiu para que surgisse uma agenda diversificada de estudos que buscavam identificar os determinantes do crescimento do PIB e a influência que os BRICS passariam a desfrutar no cenário internacional.

Levando-se em consideração que grande parte dos estudos se concentraram na análise dos fatores condicionantes do crescimento econômico desses países na primeira década dos anos 2000, o presente trabalho buscou analisar os determinantes do crescimento econômico dos BRICS no período compreendido entre 2000 a 2016. Para isso, utiliza-se do instrumental econométrico de Vetores Autorregressivos (VAR) e da Análise de Decomposição de Variância (ADV), cujos testes buscam identificar a influência de fatores domésticos (formação bruta de capital fixo, inflação, população e câmbio) e externos (fluxo de investimento direto estrangeiro e grau de abertura comercial) sobre o crescimento econômico desses países.

Estudar a dinâmica do crescimento econômico dos BRICS periodicamente torna-se importante já que são economias dotadas de enorme heterogeneidade geográfica, populacional e econômica. Ademais, o poder exercido no contexto da economia mundial requer a compreensão dos fatores que influenciam o crescimento desses países individualmente, além da sustentabilidade desse crescimento no longo prazo (OLIVEIRA; WOLF, 2016).

A pesquisa encontra-se estruturada em seis seções, incluindo esta introdução. $\mathrm{Na}$ segunda seção faz-se uma breve discussão sobre crescimento econômico, para que na terceira seção sejam apresentados os fundamentos teóricos e evidências empíricas da literatura para os BRICS. Logo depois (quarta seção) são apresentados os aspectos metodológicos da pesquisa, cujo método econométrico centra-se na utilização de dois modelos VAR de crescimento. Na quinta seção, por meio da ADV, é feita a apresentação dos dados empíricos, sendo possível, portanto, identificar a contribuição relativa de cada uma das variáveis na explicação do crescimento econômico do Brasil, Rússia, Índia, China e África do Sul. Por fim, são feitas as considerações finais do trabalho.

\section{Crescimento econômico: perspectiva teórica dos seus determinantes}

\footnotetext{
${ }^{3}$ Outros detalhamentos sobre a criação dos BRICS podem ser consultados em Oliveira e Wolf (2016).
} 
O estudo de Solow (1956) foi pioneiro na temática do crescimento econômico. Por meio do desenvolvimento de um modelo neoclássico de crescimento econômico, o autor demonstrou que a acumulação de capital, somada ao aumento da taxa de participação da força de trabalho, gerava impactos menores do que a tecnologia na explicação do crescimento do produto. Além disso, apresentou evidências de que os países com níveis baixos de renda per capita tinham tendência a desfrutar, no longo prazo, de elevadas taxas de crescimento, dado o intenso fluxo de investimentos que passariam a receber, já que o retorno do capital nas nações desenvolvidas são decrescentes e, a partir de então, os agentes buscariam melhor remuneração para o capital a ser investido.

Com base na metodologia de Solow, alguns estudos (WOO, 1996; YOUNG, 2000; KUIJS; WANG, 2005) explicam o crescimento do produto a partir das contribuições dos fatores de produção, somado ao componente residual conhecido como produtividade total dos fatores (PTF). Woo (1996), de modo particular, afirma que a acumulação de capital é a variável mais importante para a explicação das taxas de crescimento. Por sua vez, Young (2000) e Kuijs e Wang (2005) atribuem importância equivalente à acumulação de capital e produtividade do trabalho, mesmo esse último componente apresentando impacto maior em alguns períodos.

Não obstante, há estudos que buscam, fundamentalmente, investigar a contribuição relativa de um conjunto de variáveis na explicação do crescimento econômico, não se propondo a testar o modelo de crescimento de Solow. De Gregorio (1992) estudou, por meio de exercícios com dados em painel, os determinantes do crescimento econômico para 12 países latinoamericanos, no período compreendido entre 1950-1985. Constatou que a estabilidade macroeconômica e o investimento desempenham papel crucial para o crescimento de longo prazo, devendo, portanto, receber permanente atenção por parte das políticas econômicas. O consumo do governo e a instabilidade política também apresentaram alguma significância na explicação deste, porém em menor grau. Já em relação aos termos de troca, o estudo demonstrou que estes apresentam um efeito quase nulo nas taxas de crescimento.

Mesmo havendo diferenças substanciais entre os países desenvolvidos e subdesenvolvidos, particularmente em termos de renda per capita, Barro (1995) realizou uma análise com dados em painel para um conjunto de países no período compreendido entre 1960 e 1990, e demonstrou que o nível de escolaridade, expectativa de vida, baixa fertilidade, menores gastos com consumo governamental, menores taxas de inflação e melhoria nos termos de troca têm importância significativa para o crescimento econômico. Portanto, o autor destacou a 
importância de se desenvolver políticas que melhorem a infraestrutura produtiva, a relação pesquisa e desenvolvimento $(\mathrm{P} \& \mathrm{D})$ e, principalmente, a distribuição de renda e riqueza.

Borensztein, De Gregorio e Lee (1998) testaram o efeito do investimento direto estrangeiro (IDE) sobre o crescimento econômico para 69 países em desenvolvimento. Os resultados indicaram que o IDE impacta diretamente na transferência de tecnologia, contribuindo significativamente para o crescimento, sendo superior ao impacto proporcionado pelo investimento doméstico. No entanto, os referidos autores ponderaram que essa maior produtividade do IDE está intimamente relacionada com a capacidade de absorção suficiente das tecnologias avançadas por parte da nação ou região a receber o investimento, principalmente com a presença de um estoque de capital humano qualificado.

Partindo da ideia de que os países da América Latina e do Caribe têm enfrentado graves reveses em virtude das repetidas crises internacionais, reformas mal implementadas e má gestão da política macroeconômica, Loayza, Fajnzylber, Calderon (2005) empreenderam esforços no sentido de compreender os condicionantes do crescimento econômico nos países da região. Os autores destacaram que mesmo apresentando diferenças notáveis, alguns fatores estruturais como capital humano, profundidade financeira, infraestrutura pública e baixa carga tributária, além do fluxo comercial e de capital, têm relação positiva com o crescimento econômico. De modo contrário, este tende a ser desencorajado por altas e voláteis taxas de inflação, bem como pelo desalinhamento da taxa de câmbio real, indicando uma ligação entre a estabilidade macroeconômica e o crescimento de longo prazo.

Chang, Kaltani, Loayza (2009) utilizaram dados em painel para investigar a contribuição de um conjunto de variáveis sobre o crescimento econômico: abertura comercial, investimento educacional, taxas de inflação, infraestrutura e governança pública, estruturação do sistema financeiro, grau de flexibilidade do trabalho, além da dificuldade para as firmas entrarem e saírem do mercado. Os autores concluíram que o grau de abertura da economia tende a gerar impactos positivos sobre o crescimento econômico de um país. Entretanto, destacaram que a remoção de barreiras comerciais deve ser acompanhada por políticas de estabilização macroeconômica que visam aumentar a eficiência produtiva e do crescimento, o que contribuirá para atrair investimentos e proporcionar efeitos sustentados e de longo prazo.

Além dos elementos apresentados, os quais grande parte da literatura especializada os aponta como sendo importantes na determinação do crescimento econômico, verificou-se a necessidade de apresentar algumas evidências empíricas que tratam especificamente dos países que compõem os BRICS. Assim, na seção três foi feita uma síntese de alguns trabalhos que versam sobre os condicionantes do crescimento no Brasil, Rússia, Índia, China e África do Sul. 


\section{Evidências empíricas do crescimento econômico para os BRICS}

A depender do ritmo de crescimento econômico dos países do BRICS, os mesmos poderão alcançar posição privilegiada na hierarquia da economia mundial nos próximos 50 anos, conforme Wilson e Purushothaman (2003), podendo superar o grupo dos países mais desenvolvidos (Estados Unidos, Japão, Alemanha, Reino Unido, França e Itália), dado o ritmo de crescimento desses últimos em relação aos primeiros. $\mathrm{O}$

Gráfico 1 apresenta a participação dos BRICS no PIB mundial, o que demonstra a importância assumida pelo bloco no cenário mundial no período entre 2000 a 2016.

Gráfico 1 - Participação dos BRICS no PIB mundial no período de 2000 a 2016 (\%)

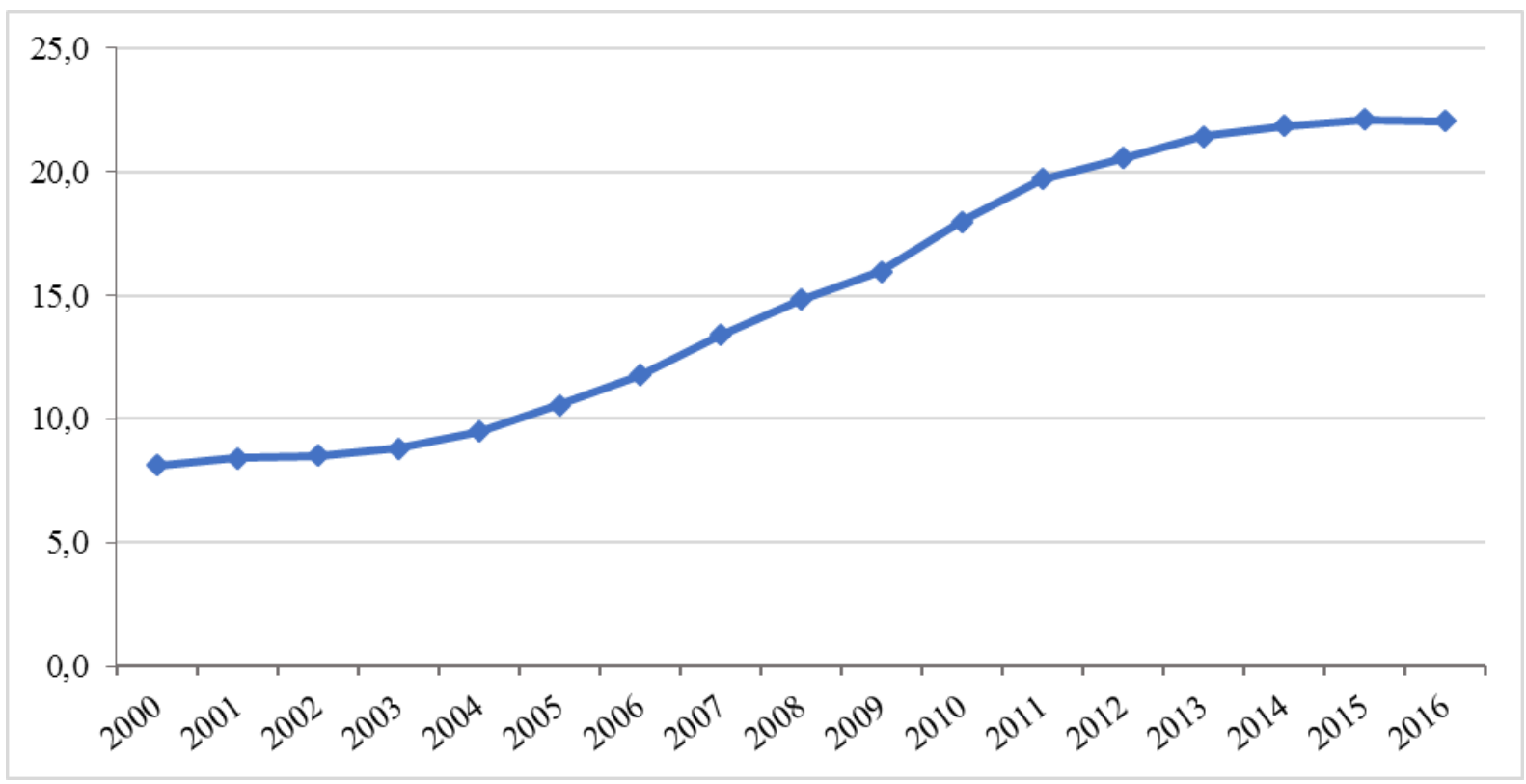

Fonte: World Bank (2019).

Em 2000 a participação dos BRICS no PIB mundial representava 8,1\%; em 2008 chegou a 14,8\% apesar da crise econômica que atingiu todas as nações. $O$ crescimento econômico contínuo desse grupo de países fez com que a participação no PIB mundial atingisse $17,9 \%$ em 2010 e 22,0\% em 2016 (Gráfico 1).

Em termos gerais, a elevada participação desse grupo de países na composição do PIB mundial tem sido decorrente das altas taxas de crescimento obtidas pela China. Conforme pode ser observado na Tabela 1, o país cresceu a uma taxa média de 10,5\% entre 2000 a 2008 e 8,3\% de 2009 a 2016. 
Tabela 1 - Taxa de crescimento econômico e populacional, segundo países dos BRICS, no período de 2000 a 2016 (\% ao ano)

BRASIL RÚSSIA ÍNDIA CHINA DO SUL

\begin{tabular}{|c|c|c|c|c|c|}
\hline \multicolumn{6}{|c|}{ Produto Interno Bruto (PIB) } \\
\hline 2000 & 4,4 & 10,0 & 3,8 & 8,5 & 4,2 \\
\hline 2008 & 5,1 & 5,2 & 3,1 & 9,7 & 3,2 \\
\hline 2009 & $-0,1$ & $-7,8$ & 7,9 & 9,4 & $-1,5$ \\
\hline 2016 & $-3,3$ & 0,3 & 8,2 & 6,7 & 0,4 \\
\hline Média de 2000 a 2008 & 3,8 & 7,0 & 6,1 & 10,5 & 4,2 \\
\hline Média de 2009 a 2016 & 3,3 & 5,0 & 7,1 & 8,3 & 3,5 \\
\hline Média de 2000 a 2016 & 4,0 & 4,9 & 6,6 & 9,4 & 3,6 \\
\hline \multicolumn{6}{|c|}{ PIB per capita } \\
\hline 2000 & 2,9 & 10,5 & 2,0 & 7,6 & 2,7 \\
\hline 2008 & 4,1 & 5,2 & 1,6 & 9,1 & 1,8 \\
\hline 2009 & $-1,1$ & $-7,8$ & 6,4 & 8,9 & $-2,9$ \\
\hline 2016 & $-4,1$ & 0,1 & 7,0 & 6,2 & $-1,1$ \\
\hline Média de 2000 a 2008 & 2,5 & 7,3 & 4,4 & 9,8 & 2,8 \\
\hline Média de 2009 a 2016 & 2,1 & 5,3 & 5,8 & 7,7 & 2,2 \\
\hline Média de 2000 a 2016 & 2,8 & 5,2 & 5,1 & 8,8 & 2,2 \\
\hline \multicolumn{6}{|c|}{ População } \\
\hline 2000 & 1,4 & $-0,4$ & 1,8 & 0,8 & 1,4 \\
\hline 2008 & 1,0 & 0,0 & 1,5 & 0,5 & 1,3 \\
\hline 2009 & 1,0 & 0,0 & 1,4 & 0,5 & 1,4 \\
\hline 2016 & 0,8 & 0,2 & 1,1 & 0,5 & 1,5 \\
\hline Média de 2000 a 2008 & 1,2 & $-0,3$ & 1,6 & 0,6 & 1,3 \\
\hline Média de 2009 a 2016 & 1,2 & $-0,3$ & 1,2 & 0,5 & 1,3 \\
\hline Média de 2000 a 2016 & 1,1 & $-0,2$ & 1,4 & 0,6 & 1,3 \\
\hline
\end{tabular}

Fonte: World Bank (2019).

Mesmo quando se considera a média de toda a série (2000-2016), a taxa de crescimento econômico da China permanece elevada ( $9,4 \%$ a.a. do PIB), refletido no crescimento do PIB per capita do país que se manteve elevado e superior aos demais integrantes do bloco (com taxa de 8,8\% de 2000 a 2016), acompanhado de relativa estabilidade do crescimento populacional. 
Além da China, é importante assinalar a contribuição da Índia no crescimento dos BRICS, principalmente por sua forte ascendência a partir de 2003, o que se refletiu em taxas de crescimento econômico, cuja variação foi, em média, de 6,0\% a 7,0\%. De acordo com Banik e Padovani (2014), o crescimento econômico da Índia está ligado ao fortalecimento das exportações de serviços tecnológicos e à consolidação de um mercado manufatureiro interno. Ademais, as possibilidades de investimentos proporcionadas pelas elevadas taxas de poupança têm contribuído para que o país alcance posição privilegiada entre as nações dos BRICS.

Já o Brasil, a Rússia e a África do Sul apresentaram taxas de crescimento econômico mais tímidas no decorrer da série analisada, inclusive com taxas negativas de crescimento em alguns anos, especialmente em 2009 quando foram atingidas pela crise econômica mundial.

No geral, nota-se que o crescimento econômico dos BRICS se deu de forma bastante desigual no período de 2000 a 2016. Essas diferenças seriam decorrentes do modelo de crescimento adotado por cada uma dessas economias, cujas diferenças se refletem fortemente no desempenho obtido ao longo do tempo. No caso particular do Brasil e a Rússia, caracterizadas por crises cambiais e de hiperinflação, notadamente nos anos de 1990, passaram a se beneficiar dos elevados preços das commodities no mercado internacional. Mesmo assim, apresentaram crescimento econômico inferior à China e Índia que têm na manufatura e no investimento estrangeiro a maior fonte de riqueza (CARNEIRO, 2002; OLIVEIRA; WOLF, 2016).

Ao analisar a formação bruta de capital fixo e a taxa de poupança interna dos BRICS, em termos de PIB, nota-se que a China e Índia são os países a apresentar o melhor desempenho em relação a essas duas variáveis. Ao analisar as Tabela 1 e Tabela 2 de forma conjunta nota-se as maiores taxas de crescimento do PIB na China e na Índia, sendo, portanto, as mesmas nações a possuírem elevadas taxas de investimento (formação bruta de capital fixo) e poupança interna bruta. Esse é um fator de grande importância apontado pela literatura como preponderante para que esses dois países tenham alcançado elevadas taxas de crescimento.

Andrade e Vieira (2007), em análise sobre a influência das variáveis tradicionais dos modelos de crescimento de Solow (investimento, renda inicial, crescimento populacional e progresso tecnológico) e outras não tradicionais (investimento, progresso tecnológico, taxa de câmbio, investimento direto estrangeiro, capital humano e grau de abertura) na explicação do crescimento econômico da China, concluíram que o investimento, progresso tecnológico e taxa de câmbio têm papel importante na explicação do crescimento econômico chinês.

De acordo com Nonnenberg (2010), o crescimento chinês é fruto de uma soma de elementos como a liberalização do sistema de formação de preços e de comércio exterior, criação de zonas econômicas especiais, crescimento dos investimentos diretos externos, explosivo 
crescimento populacional, políticas de incentivo e transferência de inovação, etc. ${ }^{4}$ Mesmo não sendo possível atribuir maior ou menor peso a uma única variável, para o autor é possível afirmar que o aumento dos gastos em investimentos, elevada taxa de poupança, elevados gastos em P\&D fazem com que a China apresente um crescimento sustentável e com condições de manter taxas similares de crescimento econômico nos próximos anos, desempenhando papel primordial para a economia mundial.

A discrepância em termos de crescimento da China e da Índia em relação aos demais países integrantes dos BRICS, em especial ao Brasil e África do Sul, se deve, em grande medida, justamente às diferenças em termos de formação bruta de capital fixo e poupança interna bruta disponível (Tabela 2).

Tabela 2 - Formação bruta de capital fixo e poupança interna bruta, segundo países dos BRICS, no período de 2000 a 2016 (\% PIB)

\begin{tabular}{llllll}
\hline PERÍODO/MÉDIA & BRASIL RÚSSIA ÍNDIA & CHINA & $\begin{array}{c}\text { ÁFRICA } \\
\text { DO SUL }\end{array}$
\end{tabular}

\begin{tabular}{lccccc}
\hline \multicolumn{5}{c}{ Formação Bruta de Capital Fixo $(F B K F)$} \\
\hline 2000 & 18,9 & 18,7 & 26,7 & 34,4 & 16,4 \\
2008 & 21,6 & 25,5 & 37,9 & 43,3 & 23,2 \\
2009 & 18,8 & 18,9 & 40,1 & 46,5 & 20,7 \\
2016 & 15,0 & 23,5 & 30,2 & 44,5 & 19,2 \\
Média de 2000 a 2008 & 18,5 & 21,5 & 33,7 & 39,8 & 18,5 \\
Média de 2009 a 2016 & 18,5 & 21,5 & 36,1 & 46,8 & 19,0 \\
Média de 2000 a 2016 & 18,8 & 21,6 & 34,8 & 43,1 & 19,4 \\
\hline & Poupança Interna Bruta & & & \\
\hline 2000 & 16,6 & 38,7 & 24,3 & 36,5 & 18,5 \\
2008 & 21,4 & 33,3 & 32,8 & 50,7 & 21,3 \\
2009 & 18,4 & 24,6 & 32,6 & 50,4 & 20,6 \\
2016 & 15,4 & 28,6 & 30,4 & 46,0 & 19,9 \\
Média de 2000 a 2008 & 19,5 & 33,6 & 29,6 & 43,9 & 19,7 \\
Média de 2009 a 2016 & 18,6 & 29,2 & 32,1 & 49,2 & 19,8 \\
Média de 2000 a 2016 & 19,1 & 31,5 & 30,8 & 46,4 & 19,8 \\
\hline
\end{tabular}

Fonte: World Bank (2019).

\footnotetext{
${ }^{4}$ Para mais informações ver Nonnenberg (2010).
} 
O investimento direto estrangeiro também tem sido apontado pela literatura especializada (OLIVEIRA; WOLF, 2016; AL-JAFARI, 2018), que trata do crescimento econômico dos BRICS, como um fator determinante para que os países integrantes desse grupo tenham alcançado altas taxas de crescimento do PIB. Conforme pode ser verificado na Tabela 3, a maior taxa de crescimento do IDE se deu na China, a saber, de 3,4\% entre 2000 e 2016, muito embora a economia chinesa apresente um grau de abertura menor do que os demais países integrantes do grupo.

Tabela 3 - Grau de abertura e investimento direto estrangeiro, segundo países dos BRICS, no período de 2000 a 2016 (\% PIB)

\begin{tabular}{llllll}
\hline PERÍODO/MÉdIA BRASIL RÚSSIA ÍNDIA CHINA & $\begin{array}{l}\text { ÁFRICA } \\
\text { DO SUL }\end{array}$
\end{tabular}

\begin{tabular}{|c|c|c|c|c|c|}
\hline \multicolumn{6}{|c|}{ Grau de Abertura } \\
\hline 2000 & 22,6 & 68,1 & 26,9 & 39,4 & 51,4 \\
\hline 2008 & 27,3 & 53,4 & 53,4 & 57,6 & 72,9 \\
\hline 2009 & 22,1 & 48,4 & 46,3 & 45,2 & 55,4 \\
\hline 2016 & 24,5 & 46,3 & 40,2 & 37,2 & 60,6 \\
\hline Média de 2000 a 2008 & 26,7 & 57,9 & 37,5 & 53,2 & 57,6 \\
\hline Média de 2009 a 2016 & 26,7 & 55,7 & 49,0 & 45,4 & 58,0 \\
\hline Média de 2000 a 2016 & 26,2 & 54,5 & 42,9 & 49,5 & 58,2 \\
\hline \multicolumn{6}{|c|}{ Investimento Direto Estrangeiro (IDE) } \\
\hline 2000 & 5,0 & 1,0 & 0,8 & 3,5 & 0,7 \\
\hline 2008 & 3,0 & 4,5 & 3,6 & 3,7 & 3,4 \\
\hline 2009 & 1,9 & 3,0 & 2,7 & 2,6 & 2,6 \\
\hline 2016 & 4,1 & 2,5 & 1,9 & 1,6 & 0,7 \\
\hline Média de 2000 a 2008 & 3,0 & 2,4 & 1,4 & 3,9 & 1,9 \\
\hline Média de 2009 a 2016 & 2,6 & 2,7 & 1,9 & 2,8 & 2,1 \\
\hline Média de 2000 a 2016 & 2,6 & 2,9 & 1,6 & 3,4 & 1,6 \\
\hline
\end{tabular}

Fonte: World Bank (2019).

Oliveira e Wolf (2016) sugerem que o investimento direto estrangeiro e o grau de abertura comercial são elementos importantes para se obter níveis de crescimento econômico de longo prazo, o que requer maior disposição do governo de cada país a adotar políticas públicas viabilizadoras desse processo. Nessa mesma perspectiva, Al-Jafari (2018) ressalta sobre a 
necessidade de que as nações empreendam esforços no sentido de remover todas as barreiras que impeçam o aporte dos investimentos estrangeiros, fonte necessária para se obter crescimento econômico mais elevado e sustentável no decorrer do tempo.

De modo similar, Vieira (2006) analisou a contribuição da taxa de investimento e grau de abertura comercial sobre o crescimento chinês. Para o autor, um conjunto de elementos internos e externos tem contribuído para o crescimento econômico do país. Dentre os fatores externos a apresentarem melhor significância ou causalidade entre as variáveis foram o investimento direto estrangeiro e o grau de abertura da economia, mas o desempenho desses indicadores depende do contexto macroeconômico interno, principalmente com taxas de inflação baixas, estabilidade cambial e pesados investimentos em capital humano.

Prabhakar et. al (2015) e Agrawal (2015) afirmam que o IDE mostrou-se preponderante na explicação das taxas de crescimento dos BRICS, de forma a ser perfeitamente perceptível a relação de longo prazo existente entre as variáveis. Nesse contexto, ressaltam a importância de se remover os obstáculos de ordem estrutural ou conjuntural que impeçam a atração e permanência desses investimentos e, assim, o país consiga desfrutar de todos os benefícios gerados por essa fonte importante de crescimento. Essa é a mesma conclusão encontrada por Vijayakumar, Sridharan e Rao (2010), materializada pela casualidade de longo prazo entre investimento direto estrangeiro e crescimento econômico, sendo necessário que cada nação implemente ações que visem a estabilidade macroeconômica de sua respectiva economia.

Tomando-se por base algumas dessas economias individualmente, alguns autores também atestam a relação em crescimento econômico e IDE. Costa et. al (2011), por exemplo, indicam que o IDE se apresentou como o principal fator na determinação das elevadas taxas de crescimento do PIB da Índia, seguido pela abertura comercial e formação bruta do capital fixo. Nesse contexto, Nassif (2006) assegura que, ressalvados os casos nos quais era permitido realizar investimento, embora fosse necessária prévia autorização, até o final da década de 1980, a economia indiana apresentava elevado grau de proteção do mercado doméstico. No entanto, diante da falta de recursos para investimento em infraestrutura de setores estratégicos, promoveuse reformas estruturais no sentido de se abrir a economia à competição de empresas privadas, mesmo sendo por meio de concessão de monopólios estatais. Diante disso, essas reformas têm contribuído significativamente para o crescimento de longo prazo do país.

Rodrik (2006) afirma que a África do Sul tem enfrentado baixa taxa de crescimento econômico em virtude das altas taxas de inflação, seguida por elevados índices de desemprego. O aludido autor afirma que tem havido o pagamento de elevados salários, sem que haja a dinamização da economia para absorção da mão de obra desempregada. Adicionalmente, a 
redução do setor não mineral e a fragilidade do setor manufatureiro voltado para as exportações, cujos índices de rentabilidade são baixos, acabam interferindo na geração de empregos e no estímulo ao crescimento econômico. A combinação de um conjunto de políticas que visem proporcionar estabilidade macroeconômica para o país torna-se importante no sentido de atrair investimentos e gerar competitividade para o seu setor exportador.

De modo geral, observadas as diferenças apresentadas por cada uma das economias integrantes dos BRICS, vários estudos atestam, portanto, a existência de correlação de longo prazo entre a dinâmica do crescimento econômico com variáveis domésticas e externas, dentre as quais destacam-se a formação bruta de capital fixo, inflação, investimento direto estrangeiro e grau de abertura. Assim, buscou-se verificar se essas variáveis permanecem com poder de explicação do crescimento econômico dos BRICS para o período de 2000 a 2016.

\section{Procedimentos metodológicos}

$\mathrm{Na}$ presente pesquisa buscou-se estimar os determinantes do crescimento econômico nas economias que compõem os BRICS, e, para isso, utilizou-se do instrumental econométrico de Vetores Autoregressivos (VAR) e Análise de Decomposição de Variância (ADV) no período compreendido entre 2000 a 2016. A escolha das variáveis se deu com base em estudos empíricos como Andrade e Vieira (2007) e Vieira e Veríssimo (2009) que tratam de modelos de crescimento econômico, sobretudo para economias emergentes. Assim, as variáveis utilizadas na pesquisa estão apresentadas no Quadro 1 - Descrição das variáveis utilizadas na análise econométrica do crescimento econômico dos BRICS.

Quadro 1 - Descrição das variáveis utilizadas na análise econométrica do crescimento econômico dos BRICS

\begin{tabular}{|l|l|l|l|}
\hline Variável & \multicolumn{1}{|c|}{ Descrição } & \multicolumn{1}{c|}{ Unidade } & \multicolumn{1}{c|}{ Fonte } \\
\hline TCPIB & Taxa de crescimento do PIB & $\begin{array}{l}\% \text { (variação } \\
\text { anual) }\end{array}$ & $\begin{array}{l}\text { World Development Indicators } \\
(2018)\end{array}$ \\
\hline FBKF & $\begin{array}{l}\text { Formação bruta de capital } \\
\text { fixo }\end{array}$ & $\%$ do PIB & $\begin{array}{l}\text { World Development Indicators } \\
(2018)\end{array}$ \\
\hline IDE & $\begin{array}{l}\text { Investimento direto } \\
\text { estrangeiro }\end{array}$ & $\%$ do PIB & $\begin{array}{l}\text { World Development Indicators } \\
(2018)\end{array}$ \\
\hline GRAB & Grau de abertura comercial & $\%$ do PIB & $\begin{array}{l}\text { World Development Indicators } \\
(2018)\end{array}$ \\
\hline
\end{tabular}




\begin{tabular}{|l|l|l|l|}
\hline POP & $\begin{array}{l}\text { Taxa de crescimento da } \\
\text { população }\end{array}$ & $\begin{array}{l}\text { \% (variação } \\
\text { anual) }\end{array}$ & $\begin{array}{l}\text { World Development Indicators } \\
(2018)\end{array}$ \\
\hline TCREF & Taxa de câmbio real efetiva & $\begin{array}{l}\text { (Índice 2010 }= \\
100)\end{array}$ & $\begin{array}{l}\text { World Development Indicators } \\
(2018)\end{array}$ \\
\hline INFLA & Taxa de inflação & $\begin{array}{l}\% \text { (variação } \\
\text { anual) }\end{array}$ & $\begin{array}{l}\text { World Development Indicators } \\
(2018)\end{array}$ \\
\hline
\end{tabular}

Fonte: Elaboração própria.

Com o intuito de examinar a contribuição relativa de determinadas variáveis no crescimento econômico dos BRICS, foram estimados dois modelos: o primeiro modelo (equação 1) levou em consideração a formação bruta de capital fixo (FBKF), o grau de abertura comercial (GRAB), a inflação (INFLA) do período, a taxa de crescimento da população (POP) e a taxa de câmbio real efetiva (TCREF). Vieira e Veríssimo (2009) destacam a importância de se incluir, para além de variáveis associadas aos modelos de crescimento econômico neoclássico, como investimento e crescimento populacional, informações que captem a estabilidade macroeconômica e grau de abertura das economias. Assim, o modelo foi especificado da seguinte forma:

$$
T C P I B=\beta_{0}+\beta_{1} F B K F+\beta_{2} \text { GRAB }+\beta_{3} I N F L+\beta_{4} P O P+\beta_{5} T C R E F+u_{t}
$$

Para o segundo modelo (equação 2), ao se incluir o investimento direto estrangeiro (IDE), em termos do produto interno bruto, optou-se por excluir a formação bruta de capital fixo (FBKF), por entendermos que esta é, em parte, explicada pela entrada de recursos oriundos do exterior. Assim, o modelo tem a seguinte especificação:

$$
T C P I B=\beta_{0}+\beta_{1} I D E+\beta_{2} G R A B+\beta_{3} I N F L+\beta_{4} P O P+\beta_{5} \text { TCREF }+u_{t}
$$

Após a escolha das variáveis, foram realizados os testes Dickey-Fuller Aumentado (ADF), Kwiatkowski-Phillips-Schmidt-Shin test statistic (KPSS) e Phillips-Perron (PP) com o intuito de detectar a estacionariedade das séries utilizadas na pesquisa para os cinco países. Aquelas que não apresentavam estacionariedade em nível foram testadas em primeira diferença, para, posteriormente, serem feitas as escolhas de defasagens (pelo critério de Schwarz) e estimações dos modelos. 
Nesse sentido, a Tabela 4 apresenta, para cada um dos países, a ordem de integração a serem utilizadas na estimação do VAR, em nível ou primeira diferença, I(0) ou I(1), respectivamente. Ademais, é apresentada a defasagem utilizada para cada economia e seus respectivos modelos que, no caso particular da presente pesquisa, só foi possível estimá-los com uma defasagem, $\operatorname{Var}(1)$, dada o número de observações da série analisada.

Tabela 4 - Ordem de integração das variáveis e defasagens do VAR

\begin{tabular}{|c|c|c|c|c|c|c|c|c|c|c|}
\hline \multirow{2}{*}{\multicolumn{2}{|c|}{ País }} & \multicolumn{7}{|c|}{ Ordem de integração } & \multicolumn{2}{|c|}{ Defasagens } \\
\hline & & TCPIB & FBKF & IDE & GRAB & INFL & POP & TCREF & $\begin{array}{c}\text { Modelo } \\
1\end{array}$ & $\begin{array}{c}\text { Modelo } \\
2\end{array}$ \\
\hline Brasil & & $\mathrm{I}(0)$ & $\mathrm{I}(1)$ & $\mathrm{I}(1)$ & $\mathrm{I}(1)$ & $\mathrm{I}(0)$ & $\mathrm{I}(0)$ & $\mathrm{I}(1)$ & $\operatorname{Var}(1)$ & $\operatorname{Var}(1)$ \\
\hline China & & $\mathrm{I}(0)$ & $\mathrm{I}(1)$ & $\mathrm{I}(1)$ & $\mathrm{I}(1)$ & $\mathrm{I}(0)$ & $\mathrm{I}(0)$ & $\mathrm{I}(0)$ & $\operatorname{Var}(1)$ & $\operatorname{Var}(1)$ \\
\hline Índia & & $\mathrm{I}(0)$ & $\mathrm{I}(1)$ & $\mathrm{I}(1)$ & $\mathrm{I}(1)$ & $\mathrm{I}(0)$ & $\mathrm{I}(1)$ & $\mathrm{I}(1)$ & $\operatorname{Var}(1)$ & $\operatorname{Var}(1)$ \\
\hline África & do & $\mathrm{I}(0)$ & $\mathrm{I}(0)$ & $\mathrm{I}(1)$ & $\mathrm{I}(1)$ & $\mathrm{I}(1)$ & $\mathrm{I}(1)$ & $\mathrm{I}(1)$ & $\operatorname{Var}(1)$ & $\operatorname{Var}(1)$ \\
\hline Sul & & & & & & & & & & \\
\hline Rússia & & $\mathrm{I}(0)$ & $\mathrm{I}(1)$ & $\mathrm{I}(1)$ & $\mathrm{I}(1)$ & $\mathrm{I}(0)$ & $\mathrm{I}(1)$ & $\mathrm{I}(1)$ & $\operatorname{Var}(1)$ & $\operatorname{Var}(1)$ \\
\hline
\end{tabular}

Fonte: Elaboração própria.

Nota: I(0) - série estacionária em nível; I(1) - série estacionária em primeira diferença.

Conforme pode-se observar na Tabela 4, apenas a TCPIB apresentou processo I(0), ou seja, estacionariedade em nível, para as quatros economias, sendo necessário realizar primeira diferença para as demais séries. Cabe destacar que todos os testes foram realizados incluindo apenas intercepto na equação.

Após essa etapa, partiu-se para a análise da ADV, cujo objetivo foi verificar o impacto relativo de cada uma das variáveis na explicação da taxa de crescimento econômico dos BRICS. Para tanto, considerando o objetivo da pesquisa, optou-se pela apresentação apenas dos dados relacionados a ADV para TCPIB nos períodos 1, 5 e 10.

\section{Resultados econométricos}

Os resultados da ADV para o Brasil (modelo 1) demonstram que o investimento e a inflação foram os principais determinantes do crescimento econômico do Brasil no período de 2000 a 2016, com 10,27\% e 8,98\%, respectivamente. Já para o modelo 2, em que se substituiu a FBKF pelo IDE, dado que a primeira é explicada, em parte, por recursos oriundos do exterior, 
verifica-se que o grau de abertura $(9,45 \%)$ e taxa de câmbio real efetiva $(9,14 \%)$ são predominantes na explicação da variação do crescimento do PIB. Se analisarmos conjuntamente o percentual dessas variáveis nos dois modelos, nota-se que, para o caso brasileiro, o investimento, a taxa de câmbio real efetiva e a inflação são determinantes para a explicação da TCPIB no período ora analisado (Tabela 5).

Tabela 5 - ADV para TCPIB - Brasil - Modelos 1 e 2 (2000 - 2016) - VAR (1)

\begin{tabular}{|c|c|c|c|c|c|c|}
\hline \multicolumn{7}{|c|}{ Modelo 1} \\
\hline PERÍODO & TCPIB & POP & $\mathrm{D}(\mathrm{GRAB})$ & $\begin{array}{c}\text { D(TCREF } \\
\text { (T) }\end{array}$ & $\mathrm{D}(\mathrm{FBKF})$ & INFLA \\
\hline 1 & 100.0000 & 0.000000 & 0.000000 & 0.000000 & 0.000000 & 0.000000 \\
\hline 5 & 71.89711 & 3.618018 & 1.522360 & 4.256394 & 10.13714 & 8.568983 \\
\hline 10 & 70.26443 & 4.770039 & 1.538942 & 4.183671 & 10.26659 & 8.976323 \\
\hline \multicolumn{7}{|c|}{ Modelo 2} \\
\hline \multirow[t]{2}{*}{ PERÍODO } & & & & & & $\mathrm{D}$ (TCREF \\
\hline & TCPIB & POP & $\mathrm{D}(\mathrm{GRAB})$ & $\mathrm{D}$ (IDE) & INFLA & ) \\
\hline 1 & 100.0000 & 0.000000 & 0.000000 & 0.000000 & 0.000000 & 0.000000 \\
\hline 5 & 76.40217 & 0.343241 & 9.558141 & 2.595925 & 1.809191 & 9.291336 \\
\hline 10 & 75.48356 & 1.067061 & 9.449406 & 2.655039 & 2.203941 & 9.140991 \\
\hline
\end{tabular}

Fonte: Elaboração própria com base em World Bank (2019).

Nota: Os modelos seguem a ordem de Cholesky.

Em relação aos resultados da ADV para a China, nota-se, pela Tabela 6, que no modelo 1, as variáveis FBKF $(15,94 \%)$ e INFLA (12,04\%) têm predominância na explicação da taxa de crescimento do PIB do país, no período de 2000 a 2016. A taxa de crescimento da população passa a ser mais significativa no modelo 2, com uma participação de 22,91\% na explicação da TCPIB. Além do componente populacional, a TCREF e o GRAB são determinantes na explicação da taxa de crescimento do PIB da China no modelo 2, com 10,51\% e 6,59\%, respectivamente.

Tabela 6 - ADV para TCPIB - China - Modelos 1 e 2 (2000 - 2016) - VAR (1)

\begin{tabular}{c|c|c|c|c|c|c}
\hline \multicolumn{7}{c}{ Modelo 1 } \\
\hline PERÍODO & TCPIB & POP & TCREF & D(GRAB) & D(FBKF) & INFLA \\
\hline 1 & 100.0000 & 0.000000 & 0.000000 & 0.000000 & 0.000000 & 0.000000
\end{tabular}




\begin{tabular}{cccccccc}
5 & 57.89008 & 5.536111 & 4.014726 & 4.251045 & 15.89505 & 12.41299 \\
10 & 58.14491 & 5.783397 & 3.816668 & 4.276469 & 15.93583 & 12.04273 \\
\hline \multicolumn{7}{c}{ Modelo 2 } \\
\hline PERÍODO & TCPIB & D(GRAB) & POP & TCREF & D(IDE) & INFLA \\
\hline 1 & 100.0000 & 0.000000 & 0.000000 & 0.000000 & 0.000000 & 0.000000 \\
5 & 57.99797 & 4.425551 & 18.50984 & 12.72596 & 0.922540 & 5.418138 \\
10 & 55.11135 & 6.586597 & 22.91023 & 10.51108 & 0.834240 & 4.046501
\end{tabular}

Fonte: Elaboração própria com base em World Bank (2019).

Nota: Os modelos seguem a ordem de Cholesky.

Ao analisar os dados de ADV para a África do Sul, nota-se, pela Tabela 7, que as variáveis GRAB (12,61\%), INFLA (10,83\%) e TCREF (8,67\%) são as mais significativas para a explicação da taxa de crescimento do PIB do país, considerando-se o modelo 1. Para o modelo 2, no qual fora substituída a variável FBKF por IDE, as mesmas variáveis do modelo 1 são predominantes para explicar a TCPIB da África do Sul, porém com percentuais menores em relação ao modelo anterior. Um ponto que merece destaque é o fato de que, nos dois modelos, a variável investimento não se apresenta como determinante do crescimento econômico do país, FBKF $(3,25 \%)$ e IDE $(0,86 \%)$, o que pode ser reflexo da instabilidade enfrentada pelo país no decorrer dos anos.

Tabela 7 - ADV para TCPIB - África do Sul - Modelos 1 e 2 (2000 - 2016) - VAR (1)

\section{Modelo 1}

\begin{tabular}{c|c|c|c|c|c|c}
\hline PERÍODO & TCPIB & D(GRAB) & POP & FBKF & D(TCREF) & D(INFLA \\
& 100.0000 & 0.000000 & 0.000000 & 0.000000 & 0.000000 & 0.000000 \\
5 & 58.46650 & 11.42315 & 8.155237 & 1.992718 & 8.725746 & 11.23666 \\
10 & 56.78284 & 12.61608 & 7.849253 & 3.251058 & 8.672634 & 10.82814 \\
\hline
\end{tabular}

Modelo 2

\begin{tabular}{c|c|c|c|c|c|c}
\hline PERÍODO & TCPIB & D(IDE) & D(TCREF) & D(GRAB) & D(INFLA) & POP \\
\hline 1 & 100.0000 & 0.000000 & 0.000000 & 0.000000 & 0.000000 & 0.000000 \\
5 & 78.93529 & 0.844003 & 3.832456 & 6.823083 & 7.793623 & 1.771548 \\
10 & 77.41632 & 0.855248 & 3.743934 & 6.595410 & 8.423830 & 2.965262
\end{tabular}

Fonte: Elaboração própria com base em World Bank (2019).

Nota: Os modelos seguem a ordem de Cholesky. 
Para a taxa de crescimento econômico da Índia, conforme Tabela 8, a Análise de Decomposição da Variância (ADV) mostra que a taxa de câmbio real efetiva (TCREF) é predominante na explicação da TCPIB nos modelos 1 e 2, com 22,47\% e 18,62\%, respectivamente. Outras variáveis aparecem com alguma significância estatística para a explicação da taxa de crescimento, como é o caso da POP (3,44\%) e FBKF (3,26\%) no modelo 1, e POP $(4,61 \%)$ e INFLA $(3,01 \%)$ no modelo 2 , mas com pouca influência na determinação do crescimento.

Tabela 8 - ADV para TCPIB - Índia - Modelos 1 e 2 (2000 - 2016) - VAR (1)

\begin{tabular}{c|c|c|c|c|c|c}
\hline \multicolumn{7}{c}{ Modelo 1 } \\
\hline PERÍODO & \multirow{2}{*}{ TCPIB } & INFLA & D(GRAB) & D(FBKF) & D(POP) & D(TCREF \\
& 100.0000 & 0.000000 & 0.000000 & 0.000000 & 0.000000 & 0.000000 \\
\hline 1 & 69.14813 & 1.376228 & 1.444628 & 2.950826 & 2.927740 & 22.15244 \\
5 & 67.66519 & 1.676345 & 1.486626 & 3.262852 & 3.440002 & 22.46898 \\
\hline
\end{tabular}

Modelo 2

\begin{tabular}{c|c|c|c|c|c|c}
\hline PERÍODO & & & & & & D(TCREF \\
& TCPIB & INFLA & D(GRAB) & D(IDE) & D(POP) & ) \\
\hline 1 & 100.0000 & 0.000000 & 0.000000 & 0.000000 & 0.000000 & 0.000000 \\
5 & 72.74644 & 2.174321 & 0.666052 & 1.337190 & 4.513465 & 18.56253 \\
10 & 71.07044 & 3.008517 & 0.675740 & 2.008254 & 4.615606 & 18.62144
\end{tabular}

Fonte: Elaboração própria com base em World Bank (2019).

Nota: Os modelos seguem a ordem de Cholesky.

No caso da Rússia, percebe-se que a taxa de crescimento populacional (POP) e a taxa de inflação (INFLA) apresentaram maiores percentuais na determinação relativa da TCPIB, com $4,13 \%$ e 3,29\%, respectivamente, no modelo 1 . No modelo 2, essas variáveis perdem importância, dando lugar ao grau de abertura (GRAB) com 5,45\% e ao investimento direto estrangeiro (2,03\%). O conjunto das variáveis explicam 12,09\% (modelo 1) e 11,75\% (modelo 2 ) concernente à taxa de crescimento do PIB da Rússia, percentual inferior aos demais países (Tabela 9). 
Tabela 9 - ADV para TCPIB - Rússia - Modelos 1 e 2 (2000 - 2016) - VAR (1)

\section{Modelo 1}

\begin{tabular}{c|c|c|c|c|c|c}
\hline $\begin{array}{c}\text { PERÍOD } \\
\text { O }\end{array}$ & TCPIB & INFLA & D(POP) & D(GRAB) & D(FBKF) & $\begin{array}{c}\text { D(TCREF } \\
\text { ) }\end{array}$ \\
\hline 1 & 100.0000 & 0.000000 & 0.000000 & 0.000000 & 0.000000 & 0.000000 \\
5 & 88.04253 & 3.203464 & 4.103878 & 1.105460 & 0.994948 & 2.549717 \\
10 & 87.90861 & 3.291019 & 4.132273 & 1.122360 & 0.995540 & 2.550198 \\
\hline
\end{tabular}

Modelo 2

\begin{tabular}{c|c|c|c|c|c|c}
\hline $\begin{array}{c}\text { PERÍOD } \\
\text { O }\end{array}$ & TCPIB & D(GRAB) & INFLA & D(IDE) & D(TCREF & D(POP) \\
\hline 1 & 100.0000 & 0.000000 & 0.000000 & 0.000000 & 0.000000 & 0.000000 \\
5 & 88.88728 & 5.197862 & 1.403349 & 1.996699 & 1.399453 & 1.115361 \\
10 & 88.54544 & 5.459559 & 1.416611 & 2.029949 & 1.403661 & 1.144781 \\
\hline
\end{tabular}

Fonte: Elaboração própria com base em World Bank (2019).

Nota: Os modelos seguem a ordem de Cholesky.

No geral, as constatações observadas para o conjunto de países integrantes dos BRICS estão de acordo com a literatura que trata dessa temática, de que o crescimento econômico desses países é determinado por um conjunto de fatores internos e externos. Sem prejuízo dessas diferenças, um ponto em comum observável é que a gestão da política macroeconômica, sobretudo com o controle da inflação e com uma taxa de câmbio real efetiva estável e competitiva, é fundamental para se atrair investimentos e contribuir para a elevação e a manutenção da taxa de crescimento desses países no longo prazo.

\section{Considerações finais}

A presente pesquisa estimou dois modelos de Vetores Autorregressivos (Var) para verificar a contribuição relativa da formação bruta de capital fixo, inflação, taxa de crescimento da população, grau de abertura comercial, taxa de câmbio real efetiva e investimento direto estrangeiro na explicação do crescimento econômico dos BRICS no período compreendido entre 2000 a 2016. Entender a dinâmica de longo prazo do crescimento econômico desses países tornase importante, dado o papel assumido pelos mesmos na economia mundial. 
A primeira constatação observada ao longo do presente trabalho é que os países integrantes dos BRICS apresentam diferenças substanciais em termos de crescimento econômico ao longo do tempo, sendo as economias chinesa e indiana as que exibem as melhores taxas de crescimento em detrimento do Brasil, Rússia e África do Sul. Muito embora esses países constituam um bloco criado no início dos anos 2000, as disparidades existentes podem sinalizar um relativo descompasso entre as estratégias adotadas por cada uma dessas economias, além de suscitar questionamentos sobre a efetividade desse grupo na condução de uma política externa e comercial coordenada.

Em relação aos resultados obtidos pela Análise da Decomposição da Variância (ADV), para o Brasil e China verificou-se, no primeiro modelo, que o investimento e a inflação apresentaram predominância na determinação do crescimento econômico. Já no segundo modelo, com substituição da formação bruta de capital fixo pelo investimento direto estrangeiro, foi possível notar que a taxa de câmbio real efetiva (Brasil e China) e um componente populacional (China) passaram a ser dominantes na explicação do crescimento do PIB. Para a Índia, o crescimento econômico é explicado, essencialmente, pela taxa de câmbio real efetiva nos dois modelos.

Já para a África do Sul, além da taxa de câmbio e do grau de abertura, o maior percentual do crescimento é explicado pela taxa de inflação. No que se refere à Rússia, percebe-se que, muito embora o conjunto das variáveis apresentem pouca significância na explicação do crescimento, os dados sinalizam que o nível populacional, a taxa de câmbio real efetiva e o investimento direto estrangeiro são capazes de influenciar em alguma medida o crescimento econômico desse país.

Os resultados aqui apresentados estão de acordo com as evidências empíricas apontadas por outros estudos nacionais e internacionais que tratam dessa temática para o Brasil e China, apesar de haver alguma variação pontual em relação a uma ou outra variável, mas perfeitamente aceitável dada as especificidades dos modelos estimados. Assim, no geral, é possível afirmar que um conjunto de políticas de investimento e de gestão macroeconômica desses países é fundamental para se promover taxas de crescimento do PIB em níveis mais elevados e sustentáveis ao longo do tempo.

O exercício aqui proposto não teve como objetivo generalizar as proposições do crescimento de um país para outro, até mesmo pelo fato de que cada um apresenta especificidades que englobam aspectos geográficas, políticos, econômicos e institucionais. Buscou-se, unicamente, identificar a participação relativa das variáveis selecionadas na explicação do crescimento econômico dos países integrantes dos BRICS. Para outros estudos a respeito do 
crescimento econômico nesses países, sugere-se a inclusão de variáveis relacionadas ao crescimento da produtividade, exportações e/ou variáveis relacionadas aos investimentos em P\&D.

\section{Referências}

AGRAWAL, G. Foreign direct investment and economic growth in BRICS economies: a panel data analysis. Journal of Economics, Business and Management, v. 3, n. 4, p. 421-424, 2015.

ANDRADE, D. C.; VIEIRA, F. V. Uma investigação teórica e empírica sobre os determinantes do crescimento econômico na China (1978 - 2003). Economia Ensaios, v. 21, n. 22, p. 87-124, 2007.

AL-JAFARI, M. K. Determinants of economic growth in BRICS countries: a panel data analysis approach. International Journal of Accounting and Financial Reporting, v. 8, n. 3, p. 29-38, 2018. DOI: https://doi.org/10.5296/ijafr.v8i3.13372.

BANIK, A.; PADOVANI, F. Índia em transformação: o novo crescimento econômico e as perspectivas pós-crise. Revista de Sociologia Política, v. 22, n. 50, p. 67-93, jun. 2014.

BARRO, R. J. Inflation and economic growth. NBER Working Paper, n. 5326, oct. 1995.

BORENSZTEIN, E., DE GREGORIO, J., LEE, J.W. How does foreign direct investment affect economic growth? Journal of International Economics, v. 45. n. 1, 115-135, 1998. DOI: https://doi.org/10.1016/S0022-1996(97)00033-0.

CARNEIRO, R. Desenvolvimento em crise: a economia brasileira no último quarto do século XX. São Paulo: Editora Unesp/IE. Unicamp, 2002.

CHANG, R.; KALTANI, L.; LOAYZA, N. Openness can be good for growth: the role of policy complementarities. Journal of Development Economics, v. 90, p. 33-49, 2009.

COSTA, C. K. F.; PASCA, N. M. C.; MENDES, C. M. D.; MASSUDA, E. M. Determinantes do crescimento econômico: evidência empírica para Brasil, China, Índia e México (1978-2006).

Perspectivas Contemporâneas, v. 6, n. 2, p. 149-175, 2011.

DE GREGORIO, J. Economic growth in Latin America. Journal of Development Economics, v. 39, p. 59-84, 1992.

KUIJS, L.; WANG, T. China's pattern of growth: moving to sustainability and reducing inequality. Washington: World Bank Policy Research, 2005 (Working paper, n. 3.767).

LOAYZA, N.; FAJNZYLBER, P.; CALDERON, C. Economic growth in Latin America and the Caribbean: stylized facts, explanations, and forecasts. The World Bank, Washington, DC, 2005.

NASSIF, A. A economia indiana no período 1950-200411 da estagnação ao crescimento acelerado: lições para o Brasil? Rio de Janeiro: BNDES, 2006. (Texto para Discussão, n. 107). 
NONNENBERG, M. J. B. China: estabilidade e crescimento econômico. Revista de Economia Política, v. 30, n. 2, p. 201-218, 2010.

OLIVEIRA, G. C.; WOLF, P. J. W. A dinâmica do investimento estrangeiro direto realizado pelos BRICS (1995-2013). Brasília: IPEA, 2016. (Texto para Discussão, n. 2.242).

O’NEILL, J. Building better global economic BRICs. Global Economics Paper, n. 66, p. 01-16, nov. 2001.

PRABHAKAR, A. C.; AZAM, M.; BAKHTYAR, B.; IBRAHIM, Y. Foreign direct investment, trade and economic growth: a new paradigm of the BRICS. Modern Applied Science, v. 9, n. 12, p. 32-42, 2015. DOI: https://doi.org/10.5539/mas.v9n12p32.

RODRIK, D. Understanding South Africa's economic puzzles. London: Centre for Economic Policy Research, 2006. (CEPR Discussion Paper, n. 5907).

SOLOW, R. M. A contribution to the theory of economic growth. Quarterly Journal of Economics, v. 70, n. 1, p. 65-94, 1956.

VIEIRA, F. V. China: crescimento econômico de longo prazo. Revista de Economia Política, v. 26, n. 3, p. 401-424, jul./set. 2006.

VIEIRA, F. V.; VERÍSSIMO, M. P. Crescimento econômico em economias emergentes selecionadas: Brasil, Rússia, Índia, China (BRIC) e África do Sul. Economia e Sociedade, v. 18, n. 3, p. 513-546, 2009.

VIJAYAKUMAR, N.; SRIDHARAN, P.; RAO, K. C. S. Determinants of FDI in BRICS countries: a panel analysis. International Journal of Business Science and Applied Management, v. 5, n. 3, p. 1-13, 2010.

WILSON, D.; PURUSHOTHAMAN, R. Dreaming with BRICs: the path to 2050. Global Economics Paper, n. 99, 2003.

WOO, W. T. Chinese economic growth: sources and prospects. California: Economics Departament University of California, 1996.

YOUNG, A. Gold into base metals: productivity growth in the people's Republic of China during the reform period. Cambridge, NBER, 2000.

WORLD BANK. World development indicators. Disponível em: http://www.worldbank.org. Acesso em: mai. 2019. 